\title{
On the Best Exponent in Markov Inequality
}

\author{
Mirosław Baran • Leokadia Białas-Cież • \\ Beata Milówka
}

Received: 9 June 2011 / Accepted: 17 May 2012 / Published online: 2 June 2012

(C) The Author(s) 2012. This article is published with open access at Springerlink.com

\begin{abstract}
Let $E$ be a compact set preserving the Markov inequality and $m(E)$ be its best exponent i.e., $m(E)$ is the infimum of all possible exponents in this inequality on $E$. It is known that $\alpha(E) \leq \frac{1}{m(E)}$ where $\alpha(E)$ is the best exponent in Hölder continuity property of the (pluri)complex Green function (with pole at infinity) of $E$. We show that if $E \subset \mathbb{C}^{N}$ (or $\mathbb{R}^{N}$ ) with $N \geq 2$ then the Markov inequality need not be fulfilled with $m(E)$. We also construct a set $E \subset \mathbb{R}^{2}$ such that the Markov inequality holds at the tip of exponential cusps composing $E$ but for the whole set $E$ we have $m(E)=$ $\infty$. Moreover, we prove that $\sup m(E)=\infty$ where the supremum is taken over all compact sets $E \subset \mathbb{R}$ preserving the Markov inequality. Finally, we prove that if $E$ is a Markov set in $\mathbb{C}$ then its image $F(E)$ under a holomorphic mapping $F$ is a Markov set too. More precisely, we prove that $m(F(E)) \leq m(E) \cdot\left(1+\max _{\partial E \cap\left\{F^{\prime}(t)=0\right\}} \operatorname{ord}_{t} F^{\prime}\right)$.
\end{abstract}

Keywords Green function • Markov inequality • Markov exponent • Best constants $\cdot$ Holomorphic mappings

Mathematics Subject Classifications $41 \mathrm{~A} 17 \cdot 41 \mathrm{~A} 44$

M. Baran · L. Białas-Cież $(\bowtie)$

Faculty of Mathematics and Computer Science, Institute of Mathematics,

Jagiellonian University, Łojasiewicza 6, 30-348 Kraków, Poland

e-mail: Leokadia.Bialas-Ciez@im.uj.edu.pl

M. Baran

e-mail: Miroslaw.Baran@im.uj.edu.pl

Beata Milówka

State Higher Vocational School in Tarnow, Institute of Mathematical and Natural Science,

Mickiewicza 8, 33-100 Tarnów, Poland

e-mail: bmilowka@wp.pl 


\section{Introduction}

Let $K \subset \mathbb{C}^{N}(N \in\{1,2, \ldots\})$ be a compact set. The pluricomplex Green function (with pole at infinity) of $K$ is defined by the formula

$$
V_{K}(z):=\sup \{u(z): u \in \mathcal{L} \text { and } u \leq 0 \text { on } K\}, \quad z \in \mathbb{C}^{N},
$$

where $\mathcal{L}$ is the family of all plurisubharmonic functions in $\mathbb{C}^{N}$ of logarithmic growth at infinity, i.e.,

$$
\mathcal{L}:=\left\{u \text { plurisubharmonic in } \mathbb{C}^{N}: u(z)-\log \|z\| \leq \mathcal{O}(1) \text { as }\|z\| \rightarrow \infty\right\}
$$

(for background information, see [9]). In the one dimensional case, $V_{K}$ coincides with the Green function $g_{K}(\cdot, \infty)$ of the unbounded component of $\hat{\mathbb{C}} \backslash K$ with logarithmic pole at infinity (as usual $\hat{\mathbb{C}}=\mathbb{C} \cup\{\infty\}$ is the Riemann sphere).

We are interested in the Hölder continuity of the (pluri)complex Green function $V_{K}$

$$
\left|V_{K}(w)-V_{K}(z)\right| \leq A|w-z|^{\alpha}
$$

with constants $A>0, \alpha \in(0,1]$ independent of $w, z \in \mathbb{C}^{N}$. By an argument due to Błocki [18, Proposition 3.5], it is sufficient to verify condition 1 only for $w \in K$ and $z \in K_{r}$ with some positive constant $r$, where

$$
K_{r}:=\left\{z \in \mathbb{C}^{N}: \operatorname{dist}(z, K) \leq r\right\} .
$$

In other words, inequality (1) is equivalent to the existence of $C>0$ such that

$$
V_{K}(z) \leq C[\operatorname{dist}(z, K)]^{\alpha} \text { for } z \in K_{1} .
$$

This property is closely related to the arrangement of the level sets of $V_{K}$ and condition (3) can be rewritten as

$$
K_{r} \subset\left\{z \in \mathbb{C}^{N}: V_{K}(z) \leq C r^{\alpha}\right\}
$$

for $r \in(0,1]$. The exponent $\alpha$ is here the essential constant. Let $\alpha(K)$ be the best exponent in inequality (3), i.e.,

$$
\alpha(K):=\sup \left\{\alpha \in(0,1]: \exists C>0 \forall z \in K_{1} \text { inequality (3) holds }\right\} .
$$

In order to estimate $\alpha(K)$, we can make use of the connection between the (pluri)complex Green function and polynomials given by

$$
V_{K}(z)=\lim _{n \rightarrow \infty} \frac{1}{n} \log \Phi_{n}(K, z),
$$

where

$$
\Phi_{n}(K, z)=\sup \left\{\frac{|P(z)|}{\|P\|_{K}}: P: \mathbb{C}^{N} \rightarrow \mathbb{C} \text { polynomial of degree } n,\left.P\right|_{K} \neq 0\right\}
$$

and $\|\cdot\|_{K}$ is the maximum norm on $K$ (see [15] or [9, Theorem 5.1.7]).

Consider the following property of the set $K$

$$
K_{1 / n^{m}} \subset\left\{z \in \mathbb{C}^{N}: \Phi_{n}(K, z) \leq M\right\}
$$


with $M, m>0$ independent of $n$, where $K_{r}$ is defined by formule (2). The set $K$ satisfies condition (6) if and only if the well known Markov inequality holds (see [11])

$$
\||\nabla P|\|_{K} \leq M(\operatorname{deg} P)^{m}\|P\|_{K},
$$

where $\nabla P:=\left(\frac{\partial P}{\partial z_{1}}, \ldots, \frac{\partial P}{\partial z_{N}}\right),|\nabla P|=\left(\sum_{j=1}^{N}\left|\frac{\partial P}{\partial z_{j}}\right|^{2}\right)^{1 / 2}$ and the positive constants $M, m$ are independent of $P$. Every set $K$ with property (7) (or equivalently (with property (6))) is called a Markov set (with exponent $m$ ). Since $|\nabla P|=\max _{\|v\|_{2}=1}\left|\sum_{j=1}^{N} v_{j} \frac{\partial P}{\partial x_{j}}\right|=$ $\max _{\|v\|_{2}=1}\left|D_{v} P\right|$, inequality (7) is equivalent to the existence of $N$ linearly independent vectors $u_{1}, \ldots, u_{N}$ and positive constants $m_{1}, \ldots, m_{N}, M_{1}, \ldots, M_{N}$ such that $\left\|D_{u_{j}} P\right\|_{K} \leq M_{j}(\operatorname{deg} P)^{m_{j}}\|P\|_{K}, \quad j=1, \ldots, N$.

The Markov exponent of a Markov set $K$ is, by definition, the best exponent in inequality (7), i.e.,

$$
m(K):=\inf \{m>0: \exists M>0 \forall P \text { inequality (7) holds }\} .
$$

If $K$ is not a Markov set, we put $m(K):=\infty$.

The notion of the Markov exponent was introduced in [2] and we refer the interested reader to this paper for further information. We can check at once that any compact set $K \subset \mathbb{C}^{N}$ has $m(K) \geq 1$ (it is sufficient to consider polynomials $P_{j, k}(z)=$ $\left(z_{j}+a_{j}\right)^{k}, j=1, \ldots, N$, where $a=\left(a_{1}, \ldots, a_{N}\right)$ is so chosen that $\left.\left\|z_{j}+a_{j}\right\|_{K} \geq 1\right)$ and $m(K)=1$ for any ball in $\mathbb{C}^{N}$ (see [15]). If $K$ is a continuum in $\mathbb{C}$, then $m(K) \in[1,2]$ (see [12]). The real case is totally different. If $K \subset \mathbb{R}^{N}=(\Re e \mathbb{C})^{N}$ then $m(K) \geq 2$ and $m(K)=2$ for any fat compact convex set $K$ (see e.g. [8]).

Due to the connection between the Markov inequality and the regularity of (pluri)complex Green's function $V_{K}$ for any compact set $K \subset \mathbb{C}^{N}$, we can find a simply relationship between the best exponents in inequalities (3) and (7). Namely, if $V_{K}$ satisfies Hölder property (3) then inequality (7) holds with any

$$
m \geq \frac{1}{\alpha}
$$

(see [16, Lemma 3]). In particular, we obtain that $\alpha(K) \leq \frac{1}{m(K)}$.

The question about the converse implication between inequalities (3) and (7) has been an open problem for many years. Moreover, up to now, it is also not known whether $V_{K}$ is always continuous for a Markov set $K \subset \mathbb{C}^{N}$. The only answer recognized is for $K \subset \mathbb{R}$ (see [5]). In this case the answer is positive. It seems that all Markov sets are non(pluri)polar but it has been proved so far only for planar compact sets [4].

However, all known examples suggest that inequalities (3) and (7) are equivalent and $\alpha(K)=\frac{1}{m(K)}($ see $[3,12,17])$. Moreover, it is easily seen that the Hölder continuity of $V_{K}$ with exponent $\alpha=1$ is equivalent to the Markov inequality with $m=1$. Indeed, by estimate (9), we can check that if inequality (3) holds with $\alpha=1$ then properties (6) and (7) are satisfied with exponent $m=1$. It appears that also 
the converse holds. Namely, for a fixed polynomial $P$ of degree $n, z \in K_{r}$ and $z_{0} \in K$ such that $\left|z-z_{0}\right| \leq r$, we have

$$
\begin{gathered}
|P(z)| \leq \sum_{j=0}^{n} \sum_{|\beta|=j, \beta \in \mathbb{Z}_{+}^{N}} \frac{1}{\beta !}\left|D^{\beta} P\left(z_{0}\right)\right|\left|z-z_{0}\right|^{j} \leq \sum_{j=0}^{n} \sum_{|\beta|=j} \frac{1}{\beta !} M^{j} n^{j}\|P\|_{K} r^{j} \\
\leq \sum_{j=0}^{n} \frac{N^{j}}{j !} M^{j} n^{j} r^{j}\|P\|_{K} \leq e^{M N n r}\|P\|_{K},
\end{gathered}
$$

which leads to condition (4) that is equivalent to inequality (3) and $\alpha=1$.

An obvious question to ask is whether the supremum in expression (5) and the infimum in formule (8) are attained or, in other words, whether condition (3) holds with $\alpha=\alpha(K)$ and whether inequality (7) is valid with $m=m(K)$ for any compact set $K$. The first question seems to be an intricate problem and remains open. We answer the second question constructing Markov sets whose Markov exponent is not attained. More precisely, for any $p \geq 1$ we construct connected compact sets $E_{p} \subset \mathbb{C}^{N}, \widetilde{E_{p}} \subset \mathbb{R}^{N}$ (for $N \geq 2$ ) such that $m\left(E_{p}\right)=p, m\left(\widetilde{E_{p}}\right)=2 p$ and we prove that inequality (7) holds neither with $m=m\left(E_{p}\right)$ for $E_{p}$ nor with $m=m\left(\widetilde{E_{p}}\right)$ for $\widetilde{E_{p}}$ (see Propositions 2.5 and 2.6). The question about a similar result in $\mathbb{C}^{1}$ is still an open problem.

Next we consider the set $E=E_{1} \cup E_{2}$, where

$$
E_{1}=\left\{(x, y) \in \mathbb{R}^{2}:|x| \leq 1,|y| \leq e^{-\frac{1}{|x|}}\right\}, E_{2}=\left\{(x, y) \in \mathbb{R}^{2}:|y| \leq 1,|x| \leq e^{-\frac{1}{|y|}}\right\}
$$

with $e^{-\frac{1}{0}}:=0 . E$ is the union of eight images of the Zerner set [20]

$$
F=\left\{(x, y) \in \mathbb{R}^{2}: x \in[0,1], y \in\left[0, e^{-\frac{1}{x}}\right]\right\}
$$

under certain isometries. It is known that a Markov inequality is satisfied at every point of $F \backslash\{(0,0)\}$ but $F$ is not a Markov set because at the tip of the exponential cusp, a Markov inequality does not hold for $F$, i.e.,

$$
\liminf _{n \rightarrow \infty} \frac{\log \left(\left|\nabla P_{n}(0,0)\right|\left\|P_{n}\right\|_{F}^{-1}\right)}{\log n}=+\infty
$$

for some polynomials $P_{n}$ of degree $n$.

In contrast, for $E$, a Markov inequality holds at the point $(0,0)$, i.e., at the tip of the exponential cusps. It is an easy consequence of the classical Markov inequality on a segment which can be applied to the cross $([-1,1] \times\{0\}) \cup(\{0\} \times[-1,1])$. Furthermore, the partial derivatives of any polynomial $P$ are uniformly bounded: $\frac{\partial P}{\partial x}$ and $\frac{\partial P}{\partial y}$ on $E_{1}$ and on $E_{2}$, respectively (cf. [1, Example 4.1]). Note that for the sets with cusps, the most intriguing points that often present a problem, are just the tips of the cusps (see $[1,7,10,19,20]$, Propositions 2.5, 2.6 below). As for $E$, the tip of the cusps does not pose any problem. Moreover, every point of $E \backslash\{(0,0)\}$ can be reached by a polynomial curve (e.g. an interval) contained in the interior of $E$ and thus (see [1]) a Markov inequality is satisfied at every point of $E$. However, $E$ is not a Markov set, in different words, $m(E)=\infty$ as will be shown in Proposition 3.1 below. It is worth additionally noting that by the analytic accessibility criterion (see [9, Proposition 5.3.12]), the Green function of $E$ is continuous in $\mathbb{C}^{2}$.

An interesting but very difficult problem is to find the precise value of the Markov exponent of an arbitrary fixed set $K$, especially if $K$ is totally disconnected. By the 
main result of [14], we can deduce that the Markov exponent of the Cantor ternary set is less than 2.94 which is not a large number. Therefore, one may ask about the existence of an upper bound for $\sup m(K)$, where the supremum is taken over all Markov sets $K$. If we consider Markov sets $K \subset \mathbb{K}^{N}(\mathbb{K}=\mathbb{R}$ or $\mathbb{C}$ ) with $N \geq 2$, we have sup $m(K)=\infty$ (see [7] or Propositions 2.5, 2.6 below). We prove that also for $N=1, \sup m(K)=\infty$, i.e., there is no bound for the Markov exponents in the case of Markov sets contained in $\mathbb{R}$ or $\mathbb{C}$. More precisely, for any $\mu>2$ we give an example of a Markov set in $\mathbb{R}$ with the Markov exponent not less than $\mu$ (Proposition 4.1). This implies that inf $\alpha(K)=0$ where $\alpha(K)$ is defined by formule (5).

Finally we consider a problem of the behaviour of $m(K)$ under holomorphic deformations $f$ of a compact set $K \subset \mathbb{C}$. We assume that $f$ is defined in a neighbourhood of the polynomial hall $\hat{K}$ of $K$. We show (Theorem 4.2) that only the zeros of $f^{\prime}$ that are lying on the boundary $\partial K$ of $K$ have an effect on the value of $m(K)$.

We now give the details and the proofs of the results mentioned above.

\section{A Class of Markov Sets in $\mathbb{C}^{2}$}

Theorem 2.1 Let $\varphi$ be a convex, increasing $\mathcal{C}^{1}$ function defined on $[0,1]$ such that $\varphi(0)=\varphi^{\prime}(0)=0, \varphi(1)=1$ and let

$$
\alpha=\liminf _{t \rightarrow 0+} \frac{\ln \varphi(t)}{\ln t}, \beta=\limsup _{t \rightarrow 0+} \frac{\ln \varphi(t)}{\ln t} .
$$

Define

$$
E=E(\varphi, \mathbb{K})=\left\{(z, w) \in \mathbb{K}^{2}:|z| \leq 1,|w| \leq \varphi(1-|z|)\right\}
$$

Then

$$
\alpha \leq m(E(\varphi, \mathbb{C})) \leq \beta
$$

and

$$
2 \alpha \leq m(E(\varphi, \mathbb{R})) \leq 2 \beta
$$

Lemma 2.2 Suppose that the function $\varphi$ satisfies the assumptions of Theorem 2.1. Then for arbitrary $s, t \in[0,1]$ we have the inequalities

$$
\begin{gathered}
\varphi(1-s t) \geq t \varphi(1-s)+\varphi(1-t) \\
1-s t-\varphi^{-1}(t \varphi(1-s)) \geq \frac{1}{\varphi^{\prime}(1)}(1-t) .
\end{gathered}
$$

Proof of Lemma 2.2 Since $\varphi$ is a $\mathcal{C}^{1}$ function then its convexity is equivalent to the fact that $\varphi^{\prime}$ is nondecreasing.

Fix $t \in[0,1]$. We need only to consider the nontrivial case $t \in(0,1)$. In such a case fix $t$ and let $f(s):=\varphi(1-s t)-\varphi(1-t)-t \varphi(1-s)$. We have $f(1)=0$ and, by the remark at the beginning of the proof,

$$
f^{\prime}(s)=-t \varphi^{\prime}(1-s t)+t \varphi^{\prime}(1-s)=t\left(\varphi^{\prime}(1-s)-\varphi^{\prime}(1-s t)\right) \leq 0 .
$$

Thus $f$ is a nonincreasing function, in particular, $f(s) \geq f(1)=0$ and inequality (12) holds. 
In order to get the second inequality, we put $\sigma:=\varphi(1-s)$. Then estimate (13) is equivalent to the inequality

$$
t \varphi^{-1}(\sigma)-\varphi^{-1}(t \sigma) \geq\left(\frac{1}{\varphi^{\prime}(1)}-1\right)(1-t) .
$$

To prove it, we fix $t \in[0,1]$ and introduce the function $g(\sigma):=t \varphi^{-1}(\sigma)-\varphi^{-1}(t \sigma)$. We check that $g^{\prime}(\sigma) \leq 0$ and therefore $g(\sigma) \geq g(1)=t-\varphi^{-1}(t)$. Finally consider the function $h(t):=t-\varphi^{-1}(t)+\left(1-\frac{1}{\varphi^{\prime}(1)}\right)(1-t)$. Since $h^{\prime}(t) \leq 0$, we have $h(t) \geq$ $h(1)=0$ and the proof is completed.

In the Proof of Theorem 2.1 we shall also need the following fact that is a complex version of the classical Schur theorem for the interval $[-1,1]$.

Proposition 2.3 (Schur's theorem for the unit disc) Let $P_{n} \in \mathbb{C}[z]$ be a polynomial of degree $n \geq 1$ such that

$$
\left|P_{n}(z)\right| \leq \frac{1}{(1-|z|)^{\gamma}}
$$

for $|z|<1$ with a positive constant $\gamma$. Then

$$
\left|P_{n}(z)\right| \leq \frac{(n+\gamma)^{n+\gamma}}{n^{n} \gamma^{\gamma}}=: C(n, \gamma)=\left(1+\frac{n}{\gamma}\right)^{\gamma}\left(1+\frac{\gamma}{n}\right)^{n}<e^{\gamma}\left(1+\frac{n}{\gamma}\right)^{\gamma}
$$

for all $|z| \leq 1$.

Moreover, this bound is sharp, because for the polynomial $P_{n}(z)=C(n, \gamma) z^{n}$ condition (14) is fulfilled and $P_{n}(1)=C(n, \gamma)$.

The above facts are equivalent to the following Schur inequality

$$
\|P\|_{\overline{\mathbb{D}}} \leq \frac{\max \left\{|P(z)|(1-|z|)^{\gamma}: z \in \overline{\mathbb{D}}\right\}}{\max \left\{|z|^{n}(1-|z|)^{\gamma}: z \in \overline{\mathbb{D}}\right\}},
$$

where $\overline{\mathbb{D}}=\overline{\mathbb{D}}_{1}$ and $\overline{\mathbb{D}}_{R}=\overline{\mathbb{D}}(0, R)$, where $\overline{\mathbb{D}}\left(z_{0}, R\right)=\left\{z \in \mathbb{C}:\left|z-z_{0}\right| \leq R\right\}$.

Proof Let $\Phi(E, z):=\lim _{n \rightarrow \infty}\left(\Phi_{n}(E, z)\right)^{\frac{1}{n}}$ be the Siciak extremal function of a compact set $E \subset \mathbb{C}^{N}$. We refer to [9] for the basic properties of this function. In particular, we have the following Bernstein-Walsh-Siciak inequality

$$
|P(z)| \leq\|P\|_{E}(\Phi(E, z))^{\operatorname{deg} P}, \quad z \in \mathbb{C}^{N} .
$$

It is well known that $\Phi\left(\overline{\mathbb{D}}_{R}, z\right)=\max \left(1, \frac{|z|}{R}\right)$. If we put $R=\frac{n}{\gamma+n}$ then, by estimates (14) and (16), we get for $z \in \mathbb{D}$

$$
|P(z)| \leq\|P\|_{\overline{\mathbb{D}}_{R}} R^{-n} \leq(1-R)^{-\gamma} R^{-n}=C(n, \gamma),
$$

which gives the first of inequalities (15). The second statement is a consequence of the easy to verify fact that $\max \left\{|z|^{n}(1-|z|)^{\gamma}:|z| \leq 1\right\}$ equals $\frac{n^{n} \gamma^{\gamma}}{(n+\gamma)^{n+\gamma}}$ and is attained for $|z|=\frac{n}{\gamma+n}$.

Proof of Theorem 2.1 Let us remark that $\varphi(t)=\varphi((1-t) 0+t \cdot 1) \leq t$ which means that $\alpha \geq 1$. We first examine the case $\mathbb{K}=\mathbb{R}$. 
The inequality $m(E(\varphi, \mathbb{R})) \leq 2 \beta$ was proved in [2]. In the same paper it was also shown that $m(E(\varphi, \mathbb{R}))=\infty$ if $\alpha=\infty$. Since $m(E) \geq 2$, it suffices to consider the case $1<\alpha<\infty$.

Let $\gamma>1$ be chosen such that $\gamma<\alpha$. Then there exists $M=M(\gamma)$ such that $\varphi(t) \leq M t^{\gamma}$. Let $l:=[2 \gamma] \in \mathbb{N}, r=\{2 \gamma\} \in[0,1)$. (Here, as usual, we denote by $[x]$ the integer number such that $x-1<[x] \leq x$ and $\{x\}=x-[x] \in[0,1)$ is the fractional part of $x$.) Then $\gamma=\frac{1}{2} l+\frac{1}{2} r$. Put

$$
P_{k}(x, y)=\left[\frac{1}{k} T_{k}^{\prime}(x)\right]^{l+1} y
$$

where $T_{k}$ denotes the $k$-th Tchebyshev polynomial (and $\frac{1}{k} T_{k}^{\prime}(x)=U_{k-1}(x)$ is the $(k-$ 1)st Tchebyshev polynomial of the second kind). Then $\operatorname{deg} P_{k}=(k-1)(l+1)+1$ and, since

$$
\left|\frac{1}{k} T_{k}^{\prime}(x)\right| \leq\left(1-x^{2}\right)^{-1 / 2} \leq(1-|x|)^{-1 / 2}, \quad x \in[-1,1],
$$

using the fact that $\left|T_{k}^{\prime}(x)\right| \leq k^{2}$ for $x \in[-1,1]$, we have

$$
\left|P_{k}(x, y)\right| \leq M\left|\frac{1}{k} T_{k}^{\prime}(x)(1-|x|)^{1 / 2}\right|^{l+r}\left|\frac{1}{k} T_{k}^{\prime}(x)\right|^{1-r} \leq M k^{1-r}
$$

for $(x, y) \in E$. Therefore

$$
\left|\frac{\partial}{\partial y} P_{k}(1,0)\right|=k^{l+1}=k^{l+r} k^{1-r} \geq \frac{1}{M} k^{2 \gamma}\left\|P_{k}\right\|_{E},
$$

which implies the estimate $m(E) \geq 2 \gamma$. Hence $m(E) \geq 2 \alpha$ and inequality (11) follows.

Now consider the case $\mathbb{K}=\mathbb{C}$.

Let $\mathbb{S}=\left\{v=\left(v_{1}, v_{2}\right) \in \mathbb{C}^{2}:\left|v_{1}\right|^{2}+\left|v_{2}\right|^{2}=1\right\}$ be the unit Euclidean sphere. For a compact subset $E$ of $\mathbb{C}^{2}, v \in \mathbb{S}$ and $u \in E$ we introduce the following distance of $u=(z, w)$ to the boundary of $E$ in direction of $v$

$$
\rho_{v}(u)=\rho_{v}\left(u, \mathbb{C}^{2} \backslash E\right)=\sup \{r: u+\zeta v \in E \text { for }|\zeta| \leq r\} .
$$

Fix a polynomial $P \in \mathbb{C}[z, w]$ of degree $n \geq 1$ with $\|P\|_{E(\varphi, \mathbb{C})}=1$. We have $D_{v} P(u)=$ $\left.\frac{\partial}{\partial \zeta} P(u+\zeta v)\right|_{\zeta=0}=Q^{\prime}(0)$, where $Q(\zeta)=P(u+\zeta v)$. By Cauchy's formula, $\left|Q^{\prime}(0)\right| \leq$ $\inf _{r>0} \frac{1}{r} \sup _{|\zeta|=r}|Q(\zeta)|$. Hence

$$
\left|D_{v} P(u)\right| \leq \inf _{r>0} \frac{1}{r} \sup _{|\zeta|=r}|P(u+\zeta v)| .
$$

The next lemma can be understood as a complex version of a property of UPC sets introduced by Pawłucki and Pleśniak and slightly modified by Baran, cf. [1, 2, 10].

Lemma 2.4 Let $\psi=\left(\psi_{1}, \psi_{2}\right): \mathbb{C} \longrightarrow \mathbb{C}^{2}$ be a polynomial mapping of degree $d=$ $\max \left(\operatorname{deg} \psi_{1}, \operatorname{deg} \psi_{2}\right) \geq 1$ such that $\psi(\mathbb{D}) \subset E \subset \mathbb{C}^{2}$ and for some $M>0, m \geq 1$

$$
\rho_{v}\left(\psi(\zeta), \mathbb{C}^{2} \backslash E\right) \geq M(1-|\zeta|)^{m}, \zeta \in \overline{\mathbb{D}},
$$

where $v \in \mathbb{S}$ is a fixed vector. 
If $P$ is a polynomial of degree $n \geq 1$ with $\|P\|_{E}=1$ then

$$
\left|D_{v} P(\psi(\zeta))\right| \leq \frac{e^{m}}{M}\left(1+\frac{(n-1) d}{m}\right)^{m} \leq \frac{(d e)^{m}}{M} n^{m}, \zeta \in \mathbb{D} .
$$

Proof of Lemma 2.4 Put $Q(\zeta)=D_{v} P(\psi(\zeta))$. Applying inequality (17) we get

$$
\begin{gathered}
|Q(\zeta)| \leq \inf _{r>0} \frac{1}{r} \sup _{|\eta|=r}|P(\psi(\zeta)+\eta v)| \leq \frac{1}{\rho_{v}(\psi(\zeta))} \sup _{|\eta|=\rho_{v}(\psi(\zeta))}|P(\psi(\zeta)+\eta v)| \\
\leq \frac{1}{\rho_{v}(\psi(\zeta))} \leq \frac{1}{M(1-|\zeta|)^{m}}
\end{gathered}
$$

and, since $\operatorname{deg} Q \leq(n-1) d$, estimate (15) yields the desired conclusion.

We proceed to prove Theorem 2.1. We can assume that $\beta<\infty$. Fix $\gamma>\beta$. Then there exists a positive constant $A=A(\gamma) \leq 1$ such that

$$
\varphi(t) \geq A t^{\gamma}, t \in[0,1] .
$$

Now we consider two special cases of $v: v=e_{1}=(1,0)$ and $v=e_{2}=(0,1)$ and $\psi(\zeta)=\psi_{(z, w)}(\zeta)=\zeta(z, w)$, where $\zeta \in \mathbb{D}, \quad(z, w) \in E(\varphi, \mathbb{C}) \backslash\{(0,0)\}$. It is easy to check that

$$
\rho_{e_{1}}(z, w)=1-|z|-\varphi^{-1}(|w|), \rho_{e_{2}}(z, w)=\varphi(1-|z|)-|w|, \quad(z, w) \in E(\varphi, \mathbb{C}),
$$

whence

$$
\rho_{e_{1}}(\psi(\zeta))=1-|\zeta||z|-\varphi^{-1}(|\zeta||w|), \rho_{e_{2}}(\psi(\zeta))=\varphi(1-|\zeta||z|)-|\zeta||w|, \quad \zeta \in \overline{\mathbb{D}} .
$$

Since $E(\varphi, \mathbb{C})=\bigcup_{\left(z_{0}, w_{0}\right) \in \partial E(\varphi, \mathbb{C})} \psi_{\left(z_{0}, w_{0}\right)}(\overline{\mathbb{D}})$ (or by the maximum principle for holomorphic functions) we can assume $|w|=\varphi(1-|z|)$. Then, by Lemma 2.2, we get the estimate

$$
\begin{gathered}
\rho_{e_{1}}(\psi(\zeta)) \geq \frac{1}{\varphi^{\prime}(1)}(1-|\zeta|), \quad \zeta \in \overline{\mathbb{D}} \\
\rho_{e_{2}}(\psi(\zeta)) \geq \varphi(1-|\zeta|) \geq A(1-|\zeta|)^{\gamma} .
\end{gathered}
$$

Applying Schur's theorem (Proposition 2.3) and Lemma 2.4 we obtain

$$
\begin{gathered}
\left|D_{e_{1}} P(\psi(\zeta))\right| \leq \varphi^{\prime}(1) e n, \quad \zeta \in \mathbb{D}, \\
\left|D_{e_{2}} P(\psi(\zeta))\right| \leq A^{-1} e^{\gamma} n^{\gamma}, \zeta \in \mathbb{D} .
\end{gathered}
$$

Consequently, the Markov inequality holds with exponent $\gamma$. Hence $m(E) \leq \beta$.

Now let $1<\alpha<\infty$ and fix $1<\gamma<\alpha$. There exists a constant $A=A(\gamma) \geq 1$ such that $\varphi(t) \leq A t^{\gamma}, t \in[0,1]$.

Consider $P=P_{k}(z, w)=z^{k} w$. We have $\left\|\frac{\partial P_{k}}{\partial w}\right\|_{E}=1$ and

$$
\begin{aligned}
& \left\|P_{k}\right\|_{E} \leq \max _{t \in[0,1]} A t^{k}(1-t)^{\gamma} \\
& =A\left(\frac{k}{\gamma+k}\right)^{k}\left(\frac{\gamma}{\gamma+k}\right)^{\gamma} \leq \frac{A}{1+\gamma} \gamma^{\gamma}(\gamma+k)^{-\gamma}=B(\gamma)(\gamma+k)^{-\gamma} .
\end{aligned}
$$


Finally we get

$$
\left\|\frac{\partial P_{k}}{\partial w}\right\|_{E} \geq \frac{1}{B(\gamma)}(k+1)^{\gamma}\left\|P_{k}\right\|_{E},
$$

which implies $m(E) \geq \alpha$, and estimate (10) is proved.

Observe that, by Taylor's formula, any convex function $\varphi \in \mathcal{C}^{k}([0,1])$ such that $\varphi(1)=1, \varphi(0)=\varphi^{\prime}(0)=\ldots=\varphi^{(k-1)}(0)=0$ and $\varphi^{(k)}(0) \neq 0$ satisfies the assumptions of Theorem 2.1 and we have $\alpha=\beta=k$.

Note that (cf. [1]), if $\varphi(t)=t\left(1+\ln \frac{1}{t}\right)^{-1}, t \in[0,1]$ and $E=E(\varphi, \mathbb{R})$, then $\alpha=$ $\beta=1$ and therefore $m(E)=2$. For $p \geq 1$, the function $\varphi_{p}(t):=\varphi\left(t^{p}\right)$ satisfies the assumptions of Theorem 2.1. Moreover, we have

$$
\lim _{t \rightarrow 0+} \frac{\ln \varphi_{p}(t)}{\ln t}=p
$$

Proposition 2.5 Let $E_{p}=E\left(\varphi_{p}, \mathbb{C}\right), p \geq 1$. Then the Markov inequality on $E_{p}$ does not hold with exponent $m\left(E_{p}\right)=p$.

Proof We use similar arguments to those given above. Consider the polynomial

$$
P_{k}(z, w)=z^{k}\left(1+p \sum_{j=1}^{k} \frac{z^{j}}{j}\right) w .
$$

One can easily check that

$$
\begin{aligned}
& \left\|P_{k}\right\|_{E_{p}} \leq \max _{|z| \leq 1}\left\{|z|^{k}\left(1+p \ln \frac{1}{1-|z|}\right) \varphi_{p}(1-|z|)\right\}=\max _{|z| \leq 1}\left\{|z|^{k}(1-|z|)^{p}\right\} \\
& \leq \frac{p^{p}}{(p+k)^{p}(1+p)}, \quad\left\|\frac{\partial P_{k}}{\partial w}\right\|_{E_{p}} \geq\left(1+p \sum_{j=1}^{k} \frac{1}{j}\right) \geq 1+p \ln (k+1) \\
& \quad \geq \frac{1+p}{p^{p}}(p+k)^{p}(1+p \ln (k+1))\left\|P_{k}\right\|_{E_{p}},
\end{aligned}
$$

which completes the proof.

Proposition 2.6 Let $\widetilde{E}_{p}=E\left(\varphi_{p}, \mathbb{R}\right), p \geq 1$. Then the Markov inequality on $\widetilde{E}_{p}$ does not hold with the exponent $m\left(\widetilde{E}_{p}\right)=2 p$.

Proof Let $l=[2 p]$. Then $l+1>2 p$. Define

$$
P_{k}(x, y)=U_{k-1}(x)^{l+1}\left(1+p \sum_{j=1}^{k} \frac{x^{j}}{j}\right) y .
$$


Applying arguments from the proof of the real case of Theorem 2.1, we obtain for $(x, y) \in \widetilde{E}_{p}$

$$
\begin{aligned}
\left|P_{k}(x, y)\right| & \leq\left|\frac{1}{k} T_{k}^{\prime}(x)\right|^{l+1}\left(1+p \sum_{j=1}^{k} \frac{|x|^{j}}{j}\right) \varphi_{p}(1-|x|) \leq\left|\frac{1}{k} T_{k}^{\prime}(x)\right|^{l+1}(1-|x|)^{p} \\
& \leq\left|\frac{1}{k} T_{k}^{\prime}(x)\right|^{l+1-2 p} \cdot\left|\frac{1}{k} T_{k}^{\prime}(x)\right|^{2 p}(1-|x|)^{p} \leq k^{l+1-2 p}
\end{aligned}
$$

and thus $\left\|P_{k}\right\|_{\widetilde{E}_{p}} \leq k^{l+1-2 p}$. Moreover,

$$
\left\|\frac{\partial P_{k}}{\partial y}\right\|_{\widetilde{E}_{p}} \geq k^{l+1}\left(1+p \sum_{j=1}^{k} \frac{1}{j}\right)=k^{2 p}\left(1+p \sum_{j=1}^{k} \frac{1}{j}\right) k^{l+1-2 p},
$$

which gives

$$
\left\|\frac{\partial P_{k}}{\partial y}\right\|_{\widetilde{E}_{p}} \geq(1+p \ln k) k^{2 p}\left\|P_{k}\right\|_{\widetilde{E}_{p}} .
$$

Corollary 2.7 For an arbitrary $p \geq 1$ and for each $N \geq 2$ there exists a compact set $E$ in $\mathbb{C}^{N}$ such that $m(E)=p$ and the Markov inequality on $E$ does not hold with exponent $p$.

Remark 2.8 Let $E_{p}=\left\{(x, y) \in \mathbb{R}^{2}: x \in[0,1], 0 \leq y \leq x^{p}\right\}$ for $p \geq 1$. It was proved by Goetgheluck [7] that $m\left(E_{p}\right)=2 p$. It was the first example of a set with a cusp for which Markov exponent was calculated. A difficult part of Goetgheluck's proof was to show that $m\left(E_{p}\right) \geq 2 p$. Actually, it can be done easily by considering the polynomials $P_{k}(x, y)=\left[\frac{1}{k} T_{k}^{\prime}(1-x)\right]^{l+1} y$ where $l=[2 p]$ with $\operatorname{deg} P_{k}=$ $(l+1)(k-1)+1$. Then $\frac{\partial P_{k}}{\partial y}(0,0)=k^{l+1}$ and $\left\|P_{k}\right\|_{E_{p}} \leq k^{1-r}$ where $r=\{2 p\}=$ $2 p-[2 p]$. This implies that $\left\|\frac{\partial P_{k}}{\partial y}\right\|_{E_{p}} \geq k^{2 p}\left\|P_{k}\right\|_{E_{p}}$ and therefore $m\left(E_{p}\right) \geq 2 p$.

\section{An Example of a Non-Markov Cuspidal Set Where the Cusp is Not the Problem}

Now we take up the set

$$
E=\left\{(x, y) \in \mathbb{R}^{2}:|x| \leq 1,|y| \leq e^{-\frac{1}{|x|}}\right\} \cup\left\{(x, y) \in \mathbb{R}^{2}:|y| \leq 1,|x| \leq e^{-\frac{1}{|y|}}\right\}
$$

with $e^{-\frac{1}{0}}:=0 . E$ is the union of eight images of the Zerner set [20]

$$
F=\left\{(x, y) \in \mathbb{R}^{2}: x \in[0,1], y \in\left[0, e^{-\frac{1}{x}}\right]\right\}
$$

under certain isometries. A pointwise Markov inequality is satisfied at every point of $F \backslash\{(0,0)\}$ but $F$ is not a Markov set, because at the tip of the exponential cusp, i.e., at the point $(0,0)$, a Markov inequality does not hold. 
Regarding $E$, the tip of the exponential cusps does not pose any problem. Namely, by the classical Markov inequality for the interval $[-1,1]$, we have

$$
|\nabla P(0,0)| \leq \sqrt{2}(\operatorname{deg} P)^{2}\|P\|_{E}
$$

for any polynomial $P$ of two variables. Moreover, a Markov inequality is satisfied at every point $(x, y)$ of $E$, because each $(x, y) \neq(0,0)$ can be attained by two perpendicular segments contained in the interior of $E$ (without $(x, y)$ if necessary). However, $E$ is not a Markov set as is shown below.

Proposition 3.1 The set E defined above is not a Markov set.

\section{Proof Put}

$$
P_{n}(x, y)=x y\left(1-x^{2}\right)^{n}\left(1-y^{2}\right)^{n}
$$

It is sufficient to prove that

$$
\liminf _{n \rightarrow \infty} \frac{\ln \left(\left\|\nabla P_{n}\right\|_{E}\left\|P_{n}\right\|_{E}^{-1}\right)}{\ln n}=+\infty
$$

We can easily check that $\left\|P_{n}\right\|_{E}=\max |x||y|\left(1-x^{2}\right)^{n}\left(1-y^{2}\right)^{n}$ where the maximum is taken over all $(x, y) \in E$ such that $|x|=e^{-\frac{1}{|y|}},|y|=e^{-\frac{1}{|x|}}$. Thus

$$
\begin{aligned}
\left\|P_{n}\right\|_{E} & =\max \left\{t e^{-\frac{1}{t}}\left(1-t^{2}\right)^{n}\left(1-e^{-\frac{2}{t}}\right)^{n}: t \in[0,1]\right\} \\
& \leq \max \left\{e^{-\frac{1}{t}}\left(1-t^{2}\right)^{n}: t \in[0,1]\right\} .
\end{aligned}
$$

Put $f(t):=e^{-\frac{1}{t}}\left(1-t^{2}\right)^{n}$. An easy computation shows that $f^{\prime}$ vanishes once in the interval $(0,1)$ and $f^{\prime}\left(\left(\frac{1}{2 n}\right)^{1 / 3}\right)<0, f^{\prime}\left(\left(\frac{1}{3 n}\right)^{1 / 3}\right)>0$. Hence for any $n>1$ we can find $b=b(n) \in(2,3)$ such that $f^{\prime}\left(\left(\frac{1}{b n}\right)^{1 / 3}\right)=0$. Therefore,

$$
\left\|P_{n}\right\|_{E} \leq f\left(\left(\frac{1}{b n}\right)^{1 / 3}\right)<e^{-\sqrt[3]{b n}}<e^{-\sqrt[3]{n}}
$$

Moreover,

$$
\begin{aligned}
\left\|\nabla P_{n}\right\|_{E} & \geq\left|\nabla P_{n}\left(\frac{1}{\sqrt{n}}, e^{-\sqrt{n}}\right)\right| \geq\left|\frac{\partial P_{n}}{\partial y}\left(\frac{1}{\sqrt{n}}, e^{-\sqrt{n}}\right)\right| \\
& =\frac{1}{\sqrt{n}}\left(1-\frac{1}{n}\right)^{n}\left(1-e^{-2 \sqrt{n}}\right)^{n-1}\left(1-e^{-2 \sqrt{n}}-2 n e^{-2 \sqrt{n}}\right)
\end{aligned}
$$

which tends to zero like $\frac{1}{\sqrt{n}}$. By the above,

$$
\liminf _{n \rightarrow \infty} \frac{\ln \left(\left\|\nabla P_{n}\right\|_{E}\left\|P_{n}\right\|_{E}^{-1}\right)}{\ln n} \geq \liminf _{n \rightarrow \infty} \frac{\ln \left(e^{\sqrt[3]{n}} n^{-1 / 2}\right)}{\ln n}=+\infty,
$$

and the proof is completed. 


\section{Markov Sets in $\mathbb{C}$}

At the beginning of this section we show that

$\sup \{m(K): K \subset \mathbb{C}$ is a Markov set $\}=\sup \{m(K): K \subset \mathbb{R}$ is a Markov set $\}=\infty$.

Recall that an analogous result in $\mathbb{C}^{N}$ and $\mathbb{R}^{N}$ with $N \geq 2$ has been obtained in [7] (or is a consequence of Propositions 2.5, 2.6 in this paper).

Proposition 4.1 Let $\mu$ be a positive number and $A=2[\mu]+12$. Then

$$
E_{\mu}=\{0\} \cup \bigcup_{k=1}^{\infty}\left[a_{k}, b_{k}\right] \text { with } b_{k}=e^{-A^{k}}, a_{k}=b_{k}^{2} \text { for } k=1,2, \ldots
$$

is a Markov set and $m\left(E_{\mu}\right) \in[\mu, \infty)$.

Proof The fact that $E_{\mu}$ is a Markov set is a consequence of Goncharov and Uzun (Markov's property of compact sets in $\mathbb{R}$ (manuscript)).

In order to prove that $m\left(E_{\mu}\right) \geq \mu$, we use the following theorem (see e.g.[6]): $A$ compact set $F \subset \mathbb{R}$ is a Markov set if and only if there exist positive constants $M, m, s$ independent of $x_{0} \in F, r \in(0,1], n \in \mathbb{N}$ and of any polynomial $P$ of degree at most $n$, such that

$$
\left|P^{\prime}\left(x_{0}\right)\right| \leq \frac{M n^{m}}{r^{s}}\|P\|_{F \cap\left[x_{0}-r, x_{0}+r\right]} .
$$

An inspection of the proof shows that if $F$ is a Markov set then inequality (19) is satisfied with every

$$
s>m(F)+5
$$

(but it can also be satisfied with some $s \leq m(F)+5$ ). Put

$$
s(F):=\inf \left\{s>0: \exists M, m>0 \quad \forall x_{0} \quad \forall r \quad \forall P \text { inequality } 19 \text { holds }\right\} .
$$

By the above, $s(F) \leq m(F)+5$.

We shall have completed the proof if we show that $\mu+5 \leq s\left(E_{\mu}\right)$. Suppose that, contrary to our claim, there exists $s \in\left(s\left(E_{\mu}\right), \mu+5\right)$. For such an $s$ inequality (19) is satisfied with $F$ replaced by $E_{\mu}$. Fix $k \in\{1,2, \ldots\}$. Take $x_{0}=0, P(x)=x, r=\frac{a_{k}}{2}$. It is easy to see that $b_{k+1}<r<a_{k}$. From estimate (19) we get

$$
1=\left|P^{\prime}\left(x_{0}\right)\right| \leq M\left(\frac{a_{k}}{2}\right)^{-s}\|P\|_{E_{\mu} \cap[0, r]}
$$

with some $M>0$ depending only on $s$. Thus

$$
1 \leq 2^{s} M a_{k}^{-s} b_{k+1}=2^{s} M e^{-A^{k}(A-2 s)} .
$$

Letting $k \rightarrow \infty$ we would have a contradiction with $s<\mu+5$.

Consequently, we have $\mu+5 \leq s\left(E_{\mu}\right) \leq m\left(E_{\mu}\right)+5$ and thus $\mu \leq m\left(E_{\mu}\right)$, which completes the proof. 
Now we consider a problem of the change of the Markov exponent under a holomorphic deformations.

Theorem 4.2 Let $E$ be a polynomially convex compact subset of $\mathbb{C}$, for which Markov's inequality is satisfied with an exponent $m$. Denote by $U$ an open neighborhood of $E$ and let $F: U \longrightarrow \mathbb{C}$ be a holomorphic mapping, that is not-constant on each component $V \subset U$ such that $V \cap E \neq \emptyset$.

Then $F(E)$ has the Markov property and the Markov inequality for $F(E)$ holds with an exponent $m_{1} \leq k \cdot m$, where

$$
k=1+\max _{t \in \partial E} \operatorname{ord}_{t} F^{\prime}
$$

and

$$
\operatorname{ord}_{t_{0}} F^{\prime}=l_{j} \text { if } \lim _{t \rightarrow t_{0}}\left(t-t_{0}\right)^{-l_{j}} F^{\prime}(t)=\alpha_{0} \neq 0
$$

In the proof of the theorem we shall use a lemma, where the assumption on the polynomial convexity is essential.

Lemma 4.3 (cf. [2], Lemma 2.2) Assume that $E$ and $F$ are as in Theorem 4.2. Let $M_{2}$ be a positive constant such that $\bigcup_{t \in E} \overline{\mathbb{D}}\left(t, M_{2}\right) \subset U$. Then

$$
|(P \circ F)(t)| \leq M_{3}\|P \circ F\|_{E} \text { provided that } \operatorname{dist}(t, E) \leq \frac{M_{2}}{n^{m}},
$$

for a positive constant $M_{3}$ independent of $P \in \mathcal{P}_{n}(\mathbb{C})$.

Proof of Theorem 4.2 Let $t_{j}$ be one of the points $\left\{t_{1}, \ldots, t_{s}\right\}=E \cap\left\{t \in U: F^{\prime}(t)=\right.$ $0\} \neq \emptyset$ (if $F^{\prime}(t) \neq 0$ on $E$ we refer to [2]). We shall consider two cases: an easy one with the assumption $t_{j} \in \operatorname{int}(E)$ and the more difficult situation where $t_{j} \in \partial E$.

Firstly assume that $t_{j} \in \operatorname{int}(E)$. Choose $r_{j}>0$ such that $\overline{\mathbb{D}}\left(t_{j}, r_{j}\right) \subset E$ and $\overline{\mathbb{D}}\left(t_{j}, r_{j}\right) \cap$ $\left(F^{\prime}\right)^{-1}(\{0\})=\left\{t_{j}\right\}$. Then each set $F\left(\partial \overline{\mathbb{D}}\left(t_{j}, r_{j}\right)\right)$ is an analytic closed curve that, by a theorem of Szegö [13, Theorem 15.3.5], admits a Markov inequality with exponent 1. Thus for a fixed polynomial $P \in \mathcal{P}_{n}(\mathbb{C})$

$$
\left\|P^{\prime}\right\|_{F\left(\partial \overline{\mathbb{D}}\left(t_{j}, r_{j}\right)\right)} \leq C_{j}\left(r_{j}\right) n\|P\|_{F\left(\partial \overline{\mathbb{D}}\left(t_{j}, r_{j}\right)\right)}
$$

and, by the maximum principle for holomorphic functions,

$$
\left\|P^{\prime}\right\|_{F\left(\overline{\mathbb{D}}\left(t_{j}, r_{j}\right)\right)} \leq M_{0} n\|P\|_{F\left(\overline{\mathbb{D}}\left(t_{j}, r_{j}\right)\right)} \leq M_{0} n\|P\|_{F(E)}, \quad M_{0}=\max _{t_{j} \in \operatorname{int}(E)} C_{j}\left(r_{j}\right)
$$

We now turn to the case $t_{j} \in \partial E$ for some $j \in\{1, \ldots, s\}$. For fixed polynomial $P \in$ $\mathcal{P}_{n}(\mathbb{C})$ and $k_{j}=1+\operatorname{ord}_{t_{j}} F^{\prime}$, we define a holomorphic function

$$
G_{P}(t)=\frac{1}{\left(t-t_{j}\right)^{k_{j}-1}}(P \circ F)^{\prime}(t), t \in U .
$$


Applying Cauchy's integral formula we get

$$
\begin{aligned}
G_{P}(t) & =\frac{1}{2 \pi i} \oint_{|\zeta-t|=\rho} \frac{1}{\left(\zeta-t_{j}\right)^{k_{j}-1}} \frac{(P \circ F)^{\prime}(\zeta)}{\zeta-t} d \zeta \\
& =\frac{1}{(2 \pi i)^{2}} \oint_{|\zeta-t|=\rho} \frac{1}{\left(\zeta-t_{j}\right)^{k_{j}-1}(\zeta-t)} \oint_{|\eta-\zeta|=\sigma} \frac{(P \circ F)(\eta)}{(\eta-\zeta)^{2}} d \eta d \zeta,
\end{aligned}
$$

for sufficiently small positive numbers $\rho$ and $\sigma$.

We shall find a bound for $\left|G_{P}(t)\right|$. We have

$$
\begin{gathered}
\left|G_{P}(t)\right| \leq\left(\frac{1}{2 \pi}\right)^{2} \cdot 2 \pi \rho \sup _{|\zeta-t|=\rho}\left\{\left|\zeta-t_{j}\right|^{-\left(k_{j}-1\right)}|\zeta-t|^{-1} \cdot 2 \pi \sigma \sup _{|\eta-\zeta|=\sigma} \frac{|(P \circ F)(\eta)|}{|\eta-\zeta|^{2}}\right\} \\
=\frac{1}{\sigma} \sup _{|\zeta-t|=\rho}\left\{\left|\zeta-t_{j}\right|^{-\left(k_{j}-1\right)} \sup _{|\eta-\zeta|=\sigma}|(P \circ F)(\eta)|\right\} .
\end{gathered}
$$

If $|\zeta-t|=\rho$ and $t \in \overline{\mathbb{D}}\left(t_{j}, \frac{\rho}{2}\right)$ then

$$
\left|\zeta-t_{j}\right|=\left|\zeta-t+t-t_{j}\right| \geq|\zeta-t|-\left|t-t_{j}\right|=\rho-\left|t-t_{j}\right| \geq \frac{\rho}{2}
$$

Thus

$$
\begin{aligned}
\left|G_{P}(t)\right| & \leq \frac{1}{\sigma}\left(\frac{\rho}{2}\right)^{-\left(k_{j}-1\right)} \sup _{|\zeta-t|=\rho}\left(\sup _{|\eta-\zeta|=\sigma}|(P \circ F)(\eta)|\right) \\
& \leq \frac{1}{\sigma}\left(\frac{\rho}{2}\right)^{-\left(k_{j}-1\right)} \sup _{\left|\eta-t_{j}\right| \leq \frac{3}{2} \rho+\sigma}|(P \circ F)(\eta)| .
\end{aligned}
$$

Taking $\sigma=\frac{3}{2} \rho$ we obtain for $\left|t-t_{j}\right| \leq \frac{\rho}{2}$

$$
\left|G_{P}(t)\right| \leq \frac{1}{3} 2^{k_{j}} \rho^{-k_{j}} \sup _{\left|t-t_{j}\right| \leq 3 \rho}|(P \circ F)(t)| .
$$

According to Lemma 4.3, for $\rho=\frac{1}{3} M_{2} n^{-m}, t \in \overline{\mathbb{D}}\left(t_{j}, \frac{\rho}{2}\right)$ we obtain

$$
\left|G_{P}(t)\right| \leq \frac{1}{3} 2^{k_{j}}\left(\frac{1}{3} M_{2} n^{-m}\right)^{-k_{j}} M_{3}\|P \circ F\|_{E}=M_{4}(j) n^{k_{j} m}\|P\|_{F(E)},
$$

where

$$
M_{4}(j)=\frac{M_{3}}{3}\left(6 M_{2}^{-1}\right)^{k_{j}} .
$$

By the assumptions, $\lim _{t \rightarrow t_{j}}\left(t-t_{j}\right)^{-\left(k_{j}-1\right)} F^{\prime}(t)=\alpha_{j} \neq 0$ and therefore, there exists an $\varepsilon_{j}>0$ such that for $t \in \overline{\mathbb{D}}\left(t_{j}, \varepsilon_{j}\right)$ we have

$$
\left|\frac{F^{\prime}(t)}{\left(t-t_{j}\right)^{k_{j}-1}}\right| \geq \frac{\left|\alpha_{j}\right|}{2}>0,
$$


hence

$$
\left|F^{\prime}(t)\right| \geq \frac{\left|\alpha_{j}\right|}{2} \cdot\left|t-t_{j}\right|^{k_{j}-1}
$$

We can assume that $\frac{1}{6} M_{2} \leq \varepsilon_{j}$. For $t \in \overline{\mathbb{D}}\left(t_{j}, \frac{1}{6} M_{2} n^{-m}\right) \backslash\left\{t_{j}\right\}$ we get

$$
\left|P^{\prime}(F(t))\right|=\left|\frac{(P \circ F)^{\prime}(t)}{F^{\prime}(t)}\right|=\left|G_{P}(t)\right| \cdot\left|\frac{\left(t-t_{j}\right)^{k_{j}-1}}{F^{\prime}(t)}\right| \leq M_{4}(j) n^{k_{j} m}\|P\|_{F(E)} \cdot \frac{2}{\left|\alpha_{j}\right|} .
$$

In addition, for $\frac{1}{6} M_{2} n^{-m} \leq\left|t-t_{j}\right| \leq \varepsilon_{j}$ we have

$$
\begin{aligned}
\left|P^{\prime}(F(t))\right| & =\left|\frac{(P \circ F)^{\prime}(t)}{F^{\prime}(t)}\right| \leq\left|(P \circ F)^{\prime}(t)\right| \cdot \frac{2}{\left|\alpha_{j}\right|} \cdot\left|t-t_{j}\right|^{-\left(k_{j}-1\right)} \\
& \leq\left|(P \circ F)^{\prime}(t)\right| \cdot \frac{2}{\left|\alpha_{j}\right|} \cdot\left(\frac{1}{6} M_{2} n^{-m}\right)^{-\left(k_{j}-1\right)} \\
& =\frac{2}{\left|\alpha_{j}\right|}\left(6 M_{2}^{-1}\right)^{\left(k_{j}-1\right)} n^{\left(k_{j}-1\right) m}\left|(P \circ F)^{\prime}(t)\right| .
\end{aligned}
$$

For suitable $\tau>0$, we have

$$
\begin{aligned}
& \left|(P \circ F)^{\prime}(t)\right| \leq \frac{1}{2 \pi} \oint_{|\zeta-t|=\tau} \frac{|(P \circ F)(\zeta)|}{|\zeta-t|^{2}}|d \zeta| \\
& \quad \leq \frac{1}{2 \pi \tau^{2}} \cdot 2 \pi \tau \cdot \sup \{|(P \circ F)(\zeta)|:|\zeta-t|=\tau\} \\
& \quad=\frac{1}{\tau} \sup \{|(P \circ F)(\zeta)|:|\zeta-t|=\tau\} .
\end{aligned}
$$

Putting $\tau=\frac{1}{12} M_{2} n^{-m}$ and using the lemma once more we obtain for $t \in E$

$$
\left|(P \circ F)^{\prime}(t)\right| \leq \frac{12}{M_{2}} n^{m} \cdot M_{3}\|P \circ F\|_{E}
$$

Finally, for $t \in E$ such that $\frac{1}{6} M_{2} n^{-m} \leq\left|t-t_{j}\right| \leq \varepsilon_{j}$ we have the estimate

$$
\left|P^{\prime}(F(t))\right| \leq \frac{4}{\left|\alpha_{j}\right|} \cdot 6^{k_{j}} M_{2}^{-k_{j}} M_{3} n^{k_{j} m}\|P\|_{F(E)} \leq M_{5}(j) n^{k_{j} m}\|P\|_{F(E)},
$$

where $M_{5}(j)=\frac{4 M_{3}}{\left|\alpha_{j}\right|} \cdot 6^{k_{j}} M_{2}^{-k_{j}}$. 
Summarizing, for $t \in E \cap \overline{\mathbb{D}}\left(t_{j}, \varepsilon_{j}\right)$, we have

$$
\left|P^{\prime}(F(t))\right| \leq M_{6}(j) n^{k_{j} m}\|P\|_{F(E)},
$$

where $M_{6}(j)=\max \left(2 M_{4}(j)\left|\alpha_{j}\right|^{-1}, M_{5}(j)\right)$.

$$
\begin{aligned}
& \text { Put } r=\min _{1 \leq j \leq s} \varepsilon_{j} . \text { For } t \in E \cap \bigcup_{j=1}^{s} \overline{\mathbb{D}}\left(t_{j}, r\right) \text { we get } \\
& \qquad P^{\prime}(F(t)) \mid \leq M_{6} n^{k m}\|P\|_{F(E)},
\end{aligned}
$$

where $M_{6}=M_{0}+\max _{1 \leq j \leq s, t_{j} \in \partial E} M_{6}(j)$.

Let $M_{7}=\sup _{t \in E \backslash E_{1}}\left|F^{\prime}(t)\right|^{-1}$. Then for $t \in E \backslash E_{1}$ we have

$$
\left|P^{\prime}(F(t))\right|=\left|\frac{(P \circ F)^{\prime}(t)}{F^{\prime}(t)}\right| \leq M_{7}\left|(P \circ F)^{\prime}(t)\right|
$$

and according to estimate (21)

$$
\left|P^{\prime}(F(t))\right| \leq \frac{12}{M_{2}} M_{3} M_{7} n^{k m}\|P\|_{F(E)} .
$$

Finally, for $M_{8}=\max \left(M_{6}, 12 M_{2}^{-1} M_{3} M_{7}\right)$ we get the inequality

$$
\left\|P^{\prime}\right\|_{F(E)} \leq M_{8} n^{k m}\|P\|_{F(E)} .
$$

Open Access This article is distributed under the terms of the Creative Commons Attribution License which permits any use, distribution, and reproduction in any medium, provided the original author(s) and the source are credited.

\section{References}

1. Baran, M.: Markov inequality on sets with polynomial parametrization. Ann. Pol. Math. 60, 69-79 (1994)

2. Baran, M., Pleśniak, W., Markov's exponent of compact sets in $\mathbb{C}^{n}$. Proc. Am. Math. Soc. 123, 2785-2791 (1995)

3. Bialas-Ciez, L.: Equivalence of Markov's property and Hölder continuity of the Green function for Cantor-type sets. East J. Approx. 1(2), 249-253 (1995)

4. Bialas-Ciez, L.: Markov Sets in $\mathbb{C}$ are not Polar. Bull. Pol. Acad. Sci. 46(1), 83-89 (1998)

5. Bialas-Ciez, L., Eggink, R.: L-regularity of Markov sets and of $m$-perfect sets in the complex plane. Constr. Approx. 27, 237-252 (2008)

6. Bos, L.P., Milman, P.D.: On Markov and Sobolev type inequalities on sets in $\mathbb{R}^{n}$. In: Topics in Polynomials in One and Several Variables and Their Applications, pp. 81-100. World Sci. Publishing, River Edge (1993)

7. Goetgheluck, P.: Inégalité de Markov dans les ensembles effilés. J. Approx. Theory 30, 149-154 (1980)

8. Goetgheluck, P.: Markov's Inequality on Locally Lipschitzian Compact Subsets of $R^{N}$ in $L^{p}$-spaces. J. Approx. Theory 49, 303-310 (1987)

9. Klimek, M.: Pluripotential Theory. Oxford Science Publications, Oxford, New York, Tokyo (1991)

10. Pawłucki, W., Pleśniak, W.: Markov's inequality and $\mathcal{C}^{\infty}$ functions on sets with polynomial cusps. Math. Ann. 275, 457-480 (1986)

11. Pleśniak, W.: Markov's inequality and the existence of an extension operator for $C^{\infty}$ functions. J. Approx. Theory 61, 106-117 (1990) 
12. Pommerenke, Ch.: On the derivative of a polynomial. Mich. Math. J. 6, 373-375 (1959)

13. Rahman, Q.I., Schmeisser, G.: Analytic Theory of Polynomials. Oxford Science Publications, Oxford (2002)

14. Ransford, T., Rostand, J.: Hölder exponents of Green's functions of Cantor sets. Comput. Methods Funct. Theory 8, 151-158 (2008)

15. Siciak, J.: Extremal plurisubharmonic functions in $\mathbb{C}^{N}$. Ann. Pol. Math. 39, 175-211 (1981)

16. Siciak, J.: Highly noncontinuable functions on polynomially convex sets. Univ. Iagello. Acta Math. 25, 95-107 (1985)

17. Siciak, J.: Compact sets in $\mathbb{R}^{n}$ admitting polynomial inequalities. Proc. Steklov Inst. Math. 203, 361-366 (1995)

18. Siciak, J.: Wiener's type sufficient conditions in $\mathbb{C}^{n}$. Univ. Iagel. Acta Math. 35, 47-74 (1997)

19. Szegö, G.: Über einen Satz von A. Markoff. Math. Z. 23, 45-61 (1925)

20. Zerner, M.: Développement en série de polynômes orthonormaux des fonctions indéfiniment différentiables. C. R. Acad. Sci. Paris Sér. 1 Math. 268, 218-220 (1969) 